\title{
FEEDING HABITS OF CHIRONOMID LARVAE (INSECTA: DIPTERA) FROM A STREAM IN THE FLORESTA DA TIJUCA, RIO DE JANEIRO, BRAZIL
}

\author{
HENRIQUES-OLIVEIRA, A. L., ${ }^{1}$ NESSIMIAN, J. L. ${ }^{1}$ and DORVILLÉ, L. F. M. ${ }^{2}$ \\ ${ }^{1}$ Departamento de Zoologia, Instituto de Biologia, Universidade Federal do Rio de Janeiro, Rio de Janeiro, Brazil \\ ${ }^{2}$ Faculdade de Formação de Professores, Universidade do Estado do Rio de Janeiro, Rio de Janeiro, Brazil \\ Correspondence to: Ana Lucia Henriques de Oliveira, Laboratório de Entomologia, Depto. de Zoologia, Instituto de \\ Biologia, Universidade Federal do Rio de Janeiro, C.P. 68044, CEP 21944-970, \\ Cidade Universitária, Rio de Janeiro, RJ, Brazil, e-mail: anahenri@biologia.ufrj.br \\ Received January 14, 2002 - Accepted April 29, 2002 - Distributed May 31, 2003
}

(With 2 figures)

\begin{abstract}
Chironomids larvae are frequently one of the most abundant and diverse groups of insects in several kinds of aquatic environments. Also, they play a major role in the aquatic food webs, representing a major link among producers and secondary consumers. This work investigates the feeding behavior of the chironomid larvae present in the Rio da Fazenda, situated in the Parque Nacional da Tijuca, Rio de Janeiro, Brazil, between August 1994 and May 1995. Algae, fungi, pollen, leaf and wood fragments, animal remains, detritus and silt were the main gut contents found in the larvae studied. The main food item ingested by the larvae was detritus, except for the Stenochironomus whose main food source was leaf and wood fragments. Tanypodinae exhibited a large quantity of animal remains of several kinds in the diet. During the period studied it was observed that the diet of 16 genera (out of 24 studied) varied. Tanypodinae had mainly coarse particulate organic matter $(>1 \mathrm{~mm})$ in the gut contents, while Chironominae and Orthocladiinae had fine particulate organic matter $(<1 \mathrm{~mm})$.
\end{abstract}

Key words: food habits, gut contents, Chironomidae, larvae.

\section{RESUMO}

\section{Hábitos alimentares das larvas de Chironomidae (Insecta: Diptera) de um riacho na Floresta da Tijuca, Rio de Janeiro, Brasil}

As larvas de Chironomidae são freqüentemente o grupo de insetos mais abundante e diversificado em vários tipos de ambientes aquáticos, apresentando importante papel nas redes tróficas das comunidades aquáticas, por constituírem efetiva ligação entre produtores e consumidores. Este trabalho analisou o comportamento alimentar das larvas de Chironomidae presentes no rio da Fazenda, localizado no Parque Nacional da Tijuca, Rio de Janeiro, Brasil, entre agosto de 1994 e maio de 1995. Os principais itens alimentares encontrados foram algas, fungos, pólen, fragmentos vegetais, restos animais, detritos e silte. O principal item alimentar ingerido pelas larvas foi detrito, exceto para Stenochironomus, cujo principal componente alimentar foram fragmentos vegetais. Os Tanypodinae também exibiram grande quantidade de restos animais de diversos grupos na dieta alimentar. Foi observada variação na dieta alimentar para 16 gêneros. Os Tanypodinae apresentaram principalmente matéria orgânica particulada grossa $(>1 \mathrm{~mm})$ no conteúdo digestivo, enquanto os Chironominae e os Orthocladiinae apresentaram matéria orgânica particulada fina $(<1 \mathrm{~mm})$.

Palavras-chave: alimentação, conteúdo estomacal, Chironomidae, larva. 


\section{INTRODUCTION}

Organic matter found in flowing waters comes from the aquatic photosynthesis and mainly from terrestrial sources (Hirabayashi \& Wotton, 1998). Both allochthonous (leaves, flowers, fruits and twigs) and autochthonous detritus support the stream biota by providing it an available food supply. Several works on aquatic insects have shown their role in the decomposition processes of allocthonous material and nutrient cycling (e.g., Cummins, 1973, 1974; Merritt et al., 1984). According to Cummins (1973), a significant portion of material cycling and energy flow in freshwater ecosystems involve various forms of organic matter processing by invertebrates.

Chironomids have an important role in the food webs of aquatic communities, representing a major link between producers and secondary consumers (Tokeshi, 1995). Chironomid larvae are opportunistic omnivorous, ingesting a wide variety of food items (Cummins \& Klug, 1979). Generally, these larvae ingest five kinds of food: algae, detritus and associated microorganisms, macrophytes, wood debris, and invertebrates (Berg, 1995).

Based upon the feeding mode, larvae can be grouped in six categories: collectors (gatherers and filterers), shredders, scrapers, and predators (engulfers and piercers) (Coffman \& Ferrington, 1996). According to the classification scheme of functional feeding groups based upon feeding mode and general detritus particle size (Cummins, 1973, 1974), food items can be grouped in the following categories: coarse particulated organic matter $(\mathrm{CPOM}->1$ $\mathrm{mm}$ ), such as leaves from the riverine vegetation and macrophytes; fine particulated organic matter (FPOM $->0.5 \mu \mathrm{m}<1 \mathrm{~mm}$ ), from a great variety of resources including CPOM fragmentation, periphyton, algae, and microorganisms; and dissolved organic matter (DOM $-<0.5 \mu \mathrm{m})$.

Therefore, shredders ingest particles larger than $1 \mathrm{~mm}$, feeding on CPOM (vascular plants, macroalgae, wood and submerged leaves) and collectors and scrapers ingest particles smaller than $1 \mathrm{~mm}$. Collectors feed on FPOM and are named this way because of the reaggregation of the small particles resulting from their ingesting activities, being either particle filterers in suspension or deposited material gatherers. Throughout all the subfamilies registered this is the most common feeding process among chironomids (Oliver, 1971).

Scrapers are especially adapted to remove firmly attached algae in the exposed surfaces placed in flowing waters, sediments, and submerged organic matter. Predators (engulfers and piercers) are commonly represented by individuals from the Tanypodinae subfamily and feed on living animal tissue, frequently higher than $1 \mathrm{~mm}$. Engulfers usually attack and ingest the whole prey or its pieces, while piercers bore the prey tissues removing their body fluids (Berg, 1995). However, it is important to recognize that most chironomids are not restricted to a single feeding mode, as also pointed out by Nessimian \& Sanseverino (1998) and Nessimian et al. (1999).

The present work aims to study and analyze the feeding habits of chironomid larvae present in the rithral section of the Rio da Fazenda (Fazenda River), in the Parque Nacional da Tijuca (Tijuca National Park), Rio de Janeiro, Brazil.

\section{MATERIALS AND METHODS}

\section{Study area}

The study area is located in a $1^{\text {st }}$ order section of the Rio da Fazenda (also called Rio Humaitá), a small stony stream that flows in the Parque $\mathrm{Na}-$ cional da Tijuca. This area is completely surrounded by the urban perimeter of the city of Rio de Janeiro (Drummond, 1997), between $22^{\circ} 55^{\prime} \mathrm{S}$ and $23^{\circ} 00^{\prime} \mathrm{S}$ and $43^{\circ} 11^{\prime} \mathrm{W}$ and $43^{\circ} 19^{\prime} \mathrm{W}$, with an area of approximately $32 \mathrm{~km}^{2}$ of tropical forests. This vegetation was greatly modified by human activities, especially coffee plantations, being the result of a reforestation program dated from the second half of the nineteenth century.

The collecting site is located at $400 \mathrm{~m}$ a.m.s.l. and the stream is on average $2 \mathrm{~m}$ wide and $20 \mathrm{~cm}$ deep. At the time of the study period the stream was almost completely covered by riverine vegetation, with little direct sunlight exposure and mosses covered many stones, thus the distinction between riffle and pool areas was not very clear. 


\section{Procedures}

Chironomid larvae were collected by means of a Surber net $\left(900 \mathrm{~cm}^{2}\right.$ area and $350 \mu \mathrm{m}$ mesh size $)$ in August (winter) and November (spring) 1994, and in February (summer) and May (autumn) 1995. The sampled larvae were preserved in $80 \%$ ethanol and sorted in morphotypes under the stereoscopic microscope. The identification up to generic level was done with the aid of the taxonomic keys of Cranston et al. (1983), Epler (1995), Pinder \& Reiss (1983), Trivinho-Strixino \& Strixino (1995) and some taxonomic descriptions.

Gut content analysis was based on the observations of the gut contents of previously slide mounted larvae, under a microscope (1000x). Monthly an average of five specimens of each taxon collected were analyzed, amounting to 440 individuals in 24 taxa (some taxa does not have specimens in all season). All food items were identified and measured by means of an eyepiece micrometer scale (400x).

The percentage of each item was estimated to the subfamilies in each season and to the genera during the period studied, as well as the maximum size (in micrometers) of the ingested particles. The arithmetic mean and the standard deviation were calculated with base on the percentage of the item ingested for all individuals of each genera.

In order to evaluate if there was a significant change in the diet of the chironomid larvae, the ChiSquare $\left(\mathrm{X}^{2}\right)$ test was used with 18 degrees of freedom for each genus, based on the relative participation of each food item in each taxon collected monthly. Significant values were accepted for $\mathrm{p}<0.05$.

\section{RESULTS}

Algae, fungi, pollen, leaf and wood fragments and fibers, animal remains, particulated organic matter (detritus), and silt were the main components found in the gut content analysis (Fig. 1). The food items registered for each genus, as well as their major contributions, can be seen in Tables 1 to 3. Detritus was the most ingested food item by the majority of the studied genera. Besides that, algae, leaf and wood fragments, and fungi were common in the majority of the Chironominae and Orthocladiinae genera (Tables 2 and 3). Stenochironomus was the only genus that did not show detritus as its major component in the diet. Instead of that, this genus presented leaf and wood fragments as its main food source (Fig. 1). The Tanypodinae diet (Table 1) also included several components of animal origin, which many times came to represent more than $50 \%$ of the diet of some genera.

The mean contributions of each food item registered for each taxon are shown in Table 4, and each subfamilies among the months studied are represented in the Fig. 2. Algae were more important in the diet of the larvae studied in the winter and autumn for Chironominae and Orthocladiinae, while leaf and wood fragments were more common in the diet of the Chironominae.

Pollen was the component that showed the smallest participation in the diets, being represented in the spring and summer. Animal remains had the highest participation in the Tanypodinae diet in individuals analyzed in November and February (Fig. 2).

The results of the Chi-square test showed that the diet of 16 genera showed some variation according to the month studied. Only Chironomus, aff. Omisus, Rheotanytarsus, Stempellinella, Tanytarsus, Chironomini sp. 1, Lopescladius, and Thienemanniella did not show variation in the diet (Table 5).

Regarding the maximum size of the food items ingested by the larvae, according to the classification proposed by Cummins (1974), the biggest particle size ingested was found in cf. Djalmabatista, mainly in the specimens analyzed in February $(2,875 \mu \mathrm{m})$, and the smallest was found in Thienemanniella, mainly in the specimens analyzed in May $(5 \mu \mathrm{m})$. According to Cummins (1974), Ablabesmyia, cf. Djalmabatista, aff. Larsia, and aff. Pentaneura, from the subfamily Tanypodinae, presented coarse particulated organic matter $(\mathrm{CPOM}>1000 \mathrm{~mm})$ in the gut contents in at least one season. Labrundinia was the only genus from this subfamily that presented fine particulate organic matter (FPOM $>0.5 \mu \mathrm{m}<1 \mathrm{~mm}$ ) in its guts contents. Chironominae and Orthocladiinae genera exhibited FPOM in its guts contents. 


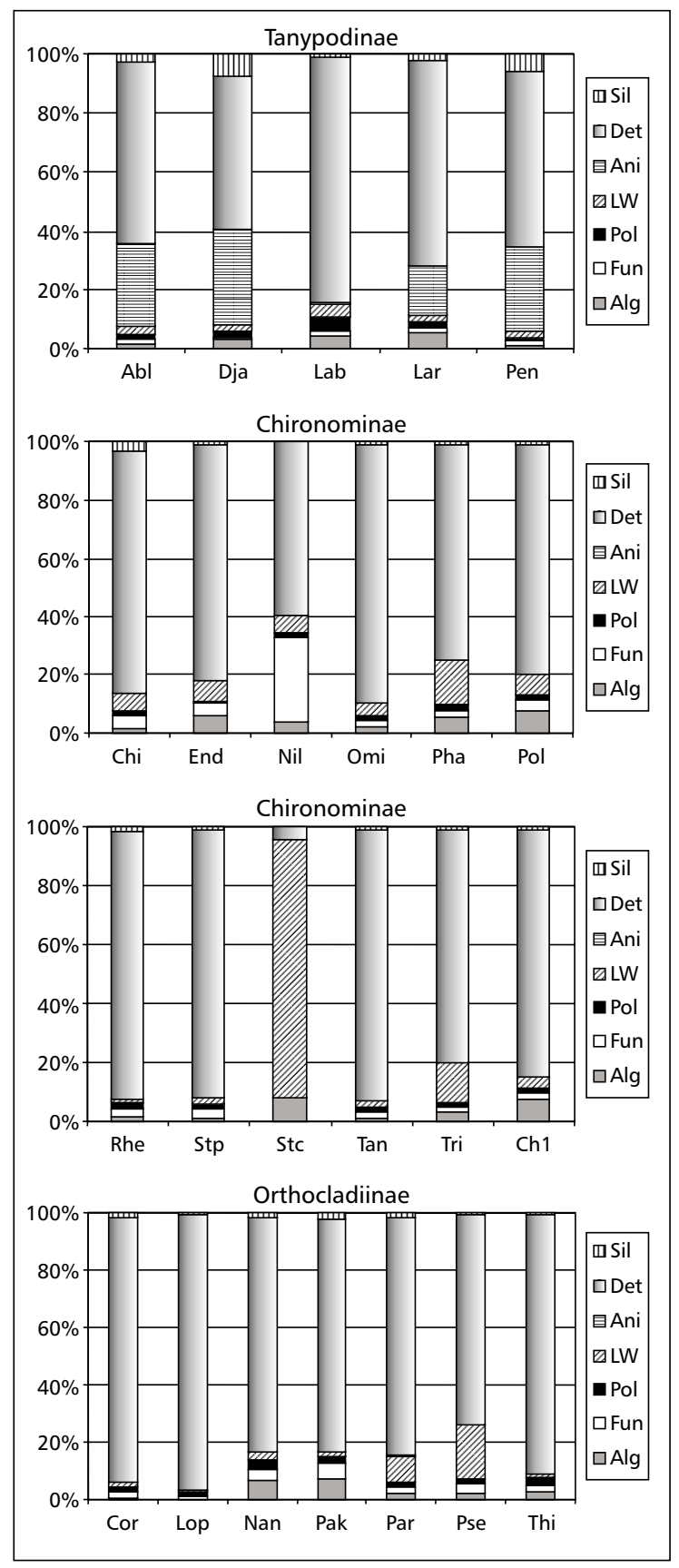

Fig. 1 - Relative composition of the food items observed in the gut contents of the chironomid larvae, for each season, in Rio da Fazenda, Parque Nacional da Tijuca, Rio de Janeiro, Brazil. Alg - algae; Fung - fungi; Pol - pollen; LW - leaf and wood fragments; Ani - animal remains; Det - detritus; Sil - silt. Abl -Ablabesmyia, Dja - cf. Djalmabatista, Lab - Labrundinia, Lar - aff. Larsia, Pen - aff. Pentaneura, Chi - Chironomus, End - Endotribelos, Nil - Nilothauma, Omi - aff. Omisus, Pha Phaenopsectra, Pol - Polypedilum, Rhe - Rheotanytarsus, Stp - Stempellinella, Stc - Stenochironomus, Tan - Tanytarsus, Tri aff. Tribelos, Ch1 - Chironomini tipo 1, Cor - Corynoneura, Lop - Lopescladius, Nan - Nanocladius, Pak - aff. Parakiefferiella, Par - Parametriocnemus, Pse - Pseudosmittia, and Thi - Thienemanniella. 
TABLE 1

Food items observed in the gut contents of members of the subfamily Tanypodinae at Rio da Fazenda, Parque Nacional da Tijuca, RJ, between August 1994 and May 1995.

\begin{tabular}{|c|c|c|c|}
\hline Taxa & $\begin{array}{c}\text { Main food items and their } \\
\text { higher percentage }\end{array}$ & Food items & Sizes \\
\hline Ablabesmyia & $\begin{array}{l}\text { Detritus }-78 \% \text { August } \\
\text { Animal remains }-48 \% \text { May }\end{array}$ & $\begin{array}{l}\text { Chlorophyceae: filamentous and } \\
\text { unicellular algae } \\
\text { Desmidiaceae (Cosmarium) } \\
\text { Fungi (conidia) } \\
\text { Leaf and wood fragments and detritus } \\
\text { Animal remains: Chironomids larvae } \\
\text { (Endotribelos, Pseudosmittia, } \\
\text { Stenochironomus), Cladocera } \\
\text { Pollens (several types) and spores }\end{array}$ & $\begin{array}{l}\text { Max. }-1050 \mu \mathrm{m} \\
\text { Min. }-50 \mu \mathrm{m}\end{array}$ \\
\hline cf. Djalmabatista & $\begin{array}{l}\text { Detritus - } 57 \% \text { November } \\
\text { and May } \\
\text { Animal remains }-50.7 \% \\
\text { February } \\
\text { Algae }-9 \% \text { August }\end{array}$ & $\begin{array}{l}\text { Chlorophyceae: filamentous, unicellular, } \\
\text { and colonial algae (Mougeotia?) } \\
\text { Desmidiaceae (Cosmarium) } \\
\text { Fungi (conidia) } \\
\text { Leaf and wood fragments and detritus } \\
\text { Animal remains: Chironomids larvae } \\
\text { (Lopescladius, Tanypodinae and } \\
\text { Tanytarsini) } \\
\text { Copepoda (nauplius), Oligochaeta setae } \\
\text { Pollens (several types) and spores }\end{array}$ & $\begin{array}{l}\text { Max. }-2875 \mu \mathrm{m} \\
\text { Min. }-50 \mu \mathrm{m}\end{array}$ \\
\hline Labrundinia & $\begin{array}{l}\text { Detritus }-90 \% \text { May } \\
\text { Pollen }-10.6 \% \text { February } \\
\text { Algae }-7 \% \text { November }\end{array}$ & $\begin{array}{l}\text { Chlorophyceae: filamentous, unicellular, } \\
\text { and colonial algae (Spyrogira) } \\
\text { Cyanophyceae (Anabaena?) } \\
\text { Desmidiaceae (Cosmarium, Staurastrum) } \\
\text { Fungi (conidia and hyphas) detritus } \\
\text { Pollens (several types) and spores }\end{array}$ & $\begin{array}{l}\text { Max. }-200 \mu \mathrm{m} \\
\text { Min. }-12.5 \mu \mathrm{m} \text { in }\end{array}$ \\
\hline aff. Larsia & $\begin{array}{l}\text { Detritus }-74.5 \% \text { May } \\
\text { Animal remains }-22.7 \% \\
\text { February } \\
\text { Algae }-14.4 \% \text { November }\end{array}$ & $\begin{array}{l}\text { Chlorophyceae: filamentous and } \\
\text { unicellular algae } \\
\text { Desmidiaceae (Cosmarium) } \\
\text { Diatomaceae } \\
\text { Fungi (conidia and hyphas), detritus } \\
\text { Animal remains: chironomid larvae, } \\
\text { Copepoda, Cladocera } \\
\text { Pollens (several types) and spores }\end{array}$ & $\begin{array}{l}\text { Max. }-1865 \mu \mathrm{m} \\
\text { Min. }-20 \mu \mathrm{m}\end{array}$ \\
\hline aff. Pentaneura & $\begin{array}{l}\text { Detritus }-69.7 \% \text { May } \\
\text { Animal remains }-46.4 \% \\
\text { November }\end{array}$ & $\begin{array}{l}\text { Chlorophyceae: filamentous, unicellular, } \\
\text { and colonial algae } \\
\text { Desmidiaceae (Cosmarium, Micrasteria) } \\
\text { Dinophyceae } \\
\text { Fungi (conidia and hyphas), detritus } \\
\text { Animal remains: Chironomids larvae } \\
\text { Copepoda, Cladocera } \\
\text { Pollens (several types) and spores }\end{array}$ & $\begin{array}{l}\text { Max. }-1912 \mu \mathrm{m} \\
\text { Min. }-25 \mu \mathrm{m}\end{array}$ \\
\hline
\end{tabular}




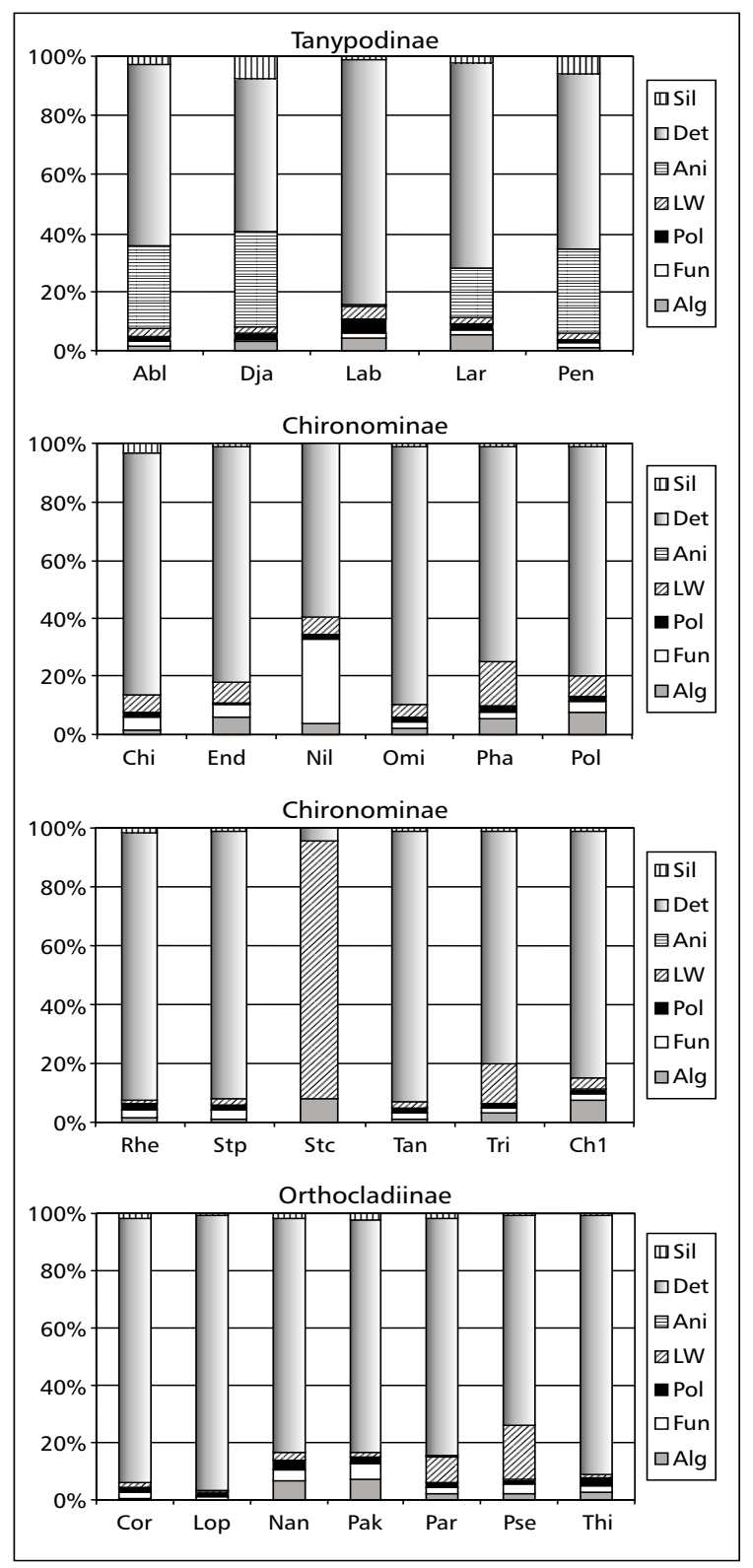

Fig. 2 - Relative composition of the food items observed in the gut contents of the chironomids subfamilies, for each season, in Rio da Fazenda, Parque Nacional da Tijuca, Rio de Janeiro, Brazil. Alg - algae; Fung - fungi; Pol - pollen; LW - leaf and wood fragments; Ani - animal remains; Det - detritus; and Sil - silt (W - winter; S - spring; Su - summer; A - autumn). 
TABLE 2

Food items observed in the gut contents of members of the subfamily chironominae at Rio da Fazenda, Parque Nacional da Tijuca, RJ, between August 1994 and May 1995.

\begin{tabular}{|c|c|c|c|}
\hline Taxa & $\begin{array}{c}\text { Main food items and their } \\
\text { higher percentage }\end{array}$ & Food items & Sizes \\
\hline Chironomus & $\begin{array}{l}\text { Detritus }-88 \% \text { November } \\
\text { Leaf and wood fragments - } \\
12.6 \% \text { February } \\
\text { Algae }-4.3 \% \text { February }\end{array}$ & $\begin{array}{l}\text { Chlorophyceae: several types } \\
\text { Cyanophyceae (Anabaena?) } \\
\text { Desmidiaceae (Micrasteria, Staurastrum) } \\
\text { Diatomaceae, Dinophyceae } \\
\text { Fungi (conidia and hyphas) } \\
\text { Leaf and wood fragments and detritus } \\
\text { Pollens (several types) and Spores }\end{array}$ & $\begin{array}{l}\text { Max. }-400 \mu \mathrm{m} \\
\text { Min. }-62 \mu \mathrm{m}\end{array}$ \\
\hline Endotribelos & $\begin{array}{l}\text { Detritus }-95.8 \% \text { May } \\
\text { Algae }-10.9 \% \text { August } \\
\text { Leaf and wood fragments - } \\
19 \% \text { August } \\
\text { Fungi }-14 \% \text { November }\end{array}$ & $\begin{array}{l}\text { Chlorophyceae: colonial and unicellular } \\
\text { algae } \\
\text { Desmidiaceae (Cosmarium) } \\
\text { Fungi (conidia and hyphas) } \\
\text { Leaf and wood fragments and detritus } \\
\text { Pollens (several types) and Spores }\end{array}$ & $\begin{array}{l}\text { Max. }-187.5 \mu \mathrm{m} \\
\text { Min. }-15 \mu \mathrm{m}\end{array}$ \\
\hline Nilothauma & $\begin{array}{l}\text { Detritus }-84 \% \text { February } \\
\text { Algae }-7 \% \text { November } \\
\text { Fungi }-100 \% \text { August } \\
\text { Leaf and wood fragments - } \\
12 \% \text { May }\end{array}$ & $\begin{array}{l}\text { Chlorophyceae: unicellular algae } \\
\text { Desmidiaceae (Micrasteria) } \\
\text { Diatomaceae } \\
\text { Fungi (hyphas) } \\
\text { Leaf and wood fragments and detritus } \\
\text { Pollens (several types) and spores }\end{array}$ & $\begin{array}{l}\text { Max. }-62.5 \mu \mathrm{m} \\
\text { Min. }-7.5 \mu \mathrm{m}\end{array}$ \\
\hline aff. Omisus & $\begin{array}{l}\text { Detritus }-90.3 \% \text { August } \\
\text { Leaf and wood fragments - } \\
5.75 \% \text { May }\end{array}$ & $\begin{array}{l}\text { Chlorophyceae: (Mougeotia?, Phycopeltis? } \\
\text { and Spyrogira); filamentous and unicellular } \\
\text { algae } \\
\text { Cyanophyceae (Anabaena?) } \\
\text { Desmidiaceae (Cosmarium) } \\
\text { Diatomaceae } \\
\text { Fungi (conidia and hyphas) } \\
\text { Leaf and wood fragments and detritus } \\
\text { Pollens (several types) }\end{array}$ & $\begin{array}{l}\text { Max. }-175 \mu \mathrm{m} \\
\text { Min. }-25 \mu \mathrm{m}\end{array}$ \\
\hline Phaenopsectra & $\begin{array}{l}\text { Detritus }-88,8 \% \text { August } \\
\text { Leaf and wood fragments - } \\
32.7 \% \text { November } \\
\text { Algae }-8.6 \% \text { November }\end{array}$ & $\begin{array}{l}\text { Chlorophyceae: (Mougeotia?) filamentous } \\
\text { and unicellular algae } \\
\text { Desmidiaceae (Cosmarium and } \\
\text { Staurastrum) } \\
\text { Diatomaceae } \\
\text { Fungi (conidia and hyphas) } \\
\text { Leaf and wood fragments and detritus } \\
\text { Pollens (several types) and spores }\end{array}$ & $\begin{array}{l}\text { Max. }-400 \mu \mathrm{m} \\
\text { Min. }-12.5 \mu \mathrm{m}\end{array}$ \\
\hline Polypedilum & $\begin{array}{l}\text { Detritus }-88 \% \text { May } \\
\text { Algae }-18,7 \% \text { August } \\
\text { Leaf and wood fragments - } \\
10 \% \text { May }\end{array}$ & $\begin{array}{l}\text { Chlorophyceae: (Mougeotia? and } \\
\text { Spyrogira) filamentous, unicellular, and } \\
\text { colonial algae } \\
\text { Cyanophyceae (Anabaena?) } \\
\text { Desmidiaceae (Cosmarium and } \\
\text { Staurastrum) } \\
\text { Diatomaceae, Dinophyceae } \\
\text { Fungi (conidia and hyphas) } \\
\text { Leaf and wood fragments and detritus } \\
\text { Pollens (several types) and spores }\end{array}$ & $\begin{array}{l}\text { Max. }-175 \mu \mathrm{m} \\
\text { Min. }-12.5 \mu \mathrm{m}\end{array}$ \\
\hline
\end{tabular}


TABLE 2 (Continued.)

\begin{tabular}{|c|c|c|c|}
\hline Taxa & $\begin{array}{l}\text { Main food items and } \\
\text { their higher percentage }\end{array}$ & Food items & Sizes \\
\hline Rheotanytarsus & $\begin{array}{l}\text { Detritus - May } 93.3 \% \\
\text { Fungi - November } 6.2 \% \\
\text { Pollen - February } 3.4 \%\end{array}$ & $\begin{array}{l}\text { Chlorophyceae: filamentous and } \\
\text { unicellular algae } \\
\text { Desmidiaceae (Cosmarium) } \\
\text { Diatomaceae } \\
\text { Fungi (conidia and hyphas) } \\
\text { Leaf and wood fragments and detritus } \\
\text { Pollens (several types) and spores }\end{array}$ & $\begin{array}{l}\text { Max. }-67.5 \mu \mathrm{m} \\
\text { Min. }-12.5 \mu \mathrm{m}\end{array}$ \\
\hline Stempellinella & $\begin{array}{l}\text { Detritus - May } 94.4 \% \\
\text { Fungi - August } 6.4 \%\end{array}$ & $\begin{array}{l}\text { Chlorophyceae: filamentous and } \\
\text { unicellular algae } \\
\text { Desmidiaceae (Micrasteria) } \\
\text { Diatomaceae } \\
\text { Fungi (conidia and hyphas) } \\
\text { Leaf and wood fragments and detritus } \\
\text { Pollens (several types) and spores }\end{array}$ & $\begin{array}{l}\text { Max. }-67.5 \mu \mathrm{m} \\
\text { Min. }-12.5 \mu \mathrm{m}\end{array}$ \\
\hline Stenochironomus & $\begin{array}{l}\text { Leaf and wood fragments - } \\
\text { February } 93.71 \% \\
\text { Algae - May } 14.5 \% \\
\text { Detritus - November } \\
15.2 \%\end{array}$ & $\begin{array}{l}\text { Chlorophyceae: filamentous, unicellular, } \\
\text { and colonial algae (Mougeotia? and } \\
\text { Spyrogira) } \\
\text { Desmidiaceae (Micrasteria) } \\
\text { Fungi (hyphas) } \\
\text { Leaf and wood fragments and detritus }\end{array}$ & $\begin{array}{l}\text { Max. }-1000 \mu \mathrm{m} \\
\text { Min. }-30 \mu \mathrm{m}\end{array}$ \\
\hline Tanytarsus & $\begin{array}{l}\text { Detritus - May } 93.6 \% \\
\text { Leaf and wood fragments - } \\
\text { August } 3.5 \%\end{array}$ & $\begin{array}{l}\text { Chlorophyceae: filamentous, unicellular, } \\
\text { and colonial algae } \\
\text { Cyanophyceae (Anabaena?) } \\
\text { Desmidiaceae (Cosmarium) } \\
\text { Fungi (conidia) } \\
\text { Leaf and wood fragments and detritus } \\
\text { Pollens (several types) and spores }\end{array}$ & $\begin{array}{l}\text { Max. }-100 \mu \mathrm{m} \\
\text { Min. }-12 \mu \mathrm{m}\end{array}$ \\
\hline aff. Tribelos & $\begin{array}{l}\text { Detritus - February } 88.3 \% \\
\text { Leaf and wood fragments - } \\
\text { May } 26.1 \% \\
\text { Fungi - November } 5.8 \% \\
\text { Algae - November } 3.6 \%\end{array}$ & $\begin{array}{l}\text { Chlorophyceae: filamentous and } \\
\text { unicellular algae (Mougeotia?; Spyrogira; } \\
\text { Phycopeltis?) } \\
\text { Desmidiaceae (Cosmarium, Micrasteria) } \\
\text { Diatomaceae } \\
\text { Fungi (conidia and hyphas) } \\
\text { Leaf and wood fragments and detritus } \\
\text { Pollens (several types) }\end{array}$ & $\begin{array}{l}\text { Max. }-475 \mu \mathrm{m} \\
\text { Min. }-25 \mu \mathrm{m}\end{array}$ \\
\hline Chironomini sp. 1 & $\begin{array}{l}\text { Detritus - February } 90.6 \% \\
\text { Algae - August } 14.8 \% \\
\text { Leaf and wood fragments - } \\
\text { August } 4.3 \%\end{array}$ & $\begin{array}{l}\text { Chlorophyceae: filamentous and } \\
\text { unicellular algae (Spyrogira) } \\
\text { Desmidiaceae (Cosmarium, Micrasteria } \\
\text { and Staurastrum) } \\
\text { Diatomaceae } \\
\text { Fungi (conidia and hyphas) } \\
\text { Leaf and wood fragments and detritus } \\
\text { Pollens (several types) and spores }\end{array}$ & $\begin{array}{l}\text { Max. }-100 \mu \mathrm{m} \\
\text { Min. }-12.5 \mu \mathrm{m}\end{array}$ \\
\hline
\end{tabular}


TABLE 3

Food habit observed in the gut contents of members of the subfamily Orthocladiinae at Rio da Fazenda, Parque Nacional da Tijuca, RJ, between August 1994 and May 1995.

\begin{tabular}{|c|c|c|c|}
\hline Taxa & $\begin{array}{c}\text { Main food items and } \\
\text { their higher percentage }\end{array}$ & Food items & Sizes \\
\hline Corynoneura & $\begin{array}{l}\text { Detritus }-97 \% \text { February } \\
\text { Fungi }-5 \% \text { August } \\
\text { Leaf and wood } \\
\text { fragments }-5 \% \text { August }\end{array}$ & $\begin{array}{l}\text { Chlorophyceae: unicellular algae } \\
\text { Fungi (conidia and hyphas) } \\
\text { Pollens (several types) }\end{array}$ & $\begin{array}{l}\text { Max. }-75 \mu \mathrm{m} \\
\text { Min. }-12.5 \mu \mathrm{m}\end{array}$ \\
\hline Lopescladius & $\begin{array}{l}\text { Detritus }-97 \% \text { August } \\
\text { Pollen }-2.5 \% \text { February }\end{array}$ & $\begin{array}{l}\text { Fungi (conidia and hyphas) } \\
\text { Leaf and wood fragments and detritus } \\
\text { Pollens (several types) }\end{array}$ & $\begin{array}{l}\text { Max. }-31.25 \mu \mathrm{m} \\
\text { Min. }-12.5 \mu \mathrm{m}\end{array}$ \\
\hline Nanocladius & $\begin{array}{l}\text { Detritus }-89.7 \% \text { February } \\
\text { Algae }-15.7 \% \text { August } \\
\text { Fungi }-12 \% \text { August } \\
\text { Pollen }-3.3 \% \text { February }\end{array}$ & $\begin{array}{l}\text { Chlorophyceae: filamentous and } \\
\text { colonial algae } \\
\text { Diatomaceae } \\
\text { Fungi (conidia and hyphas) } \\
\text { Leaf and wood fragments and detritus } \\
\text { Pollens (several types) and spores }\end{array}$ & $\begin{array}{l}\text { Max. }-90 \mu \mathrm{m} \\
\text { Min. }-12.5 \mu \mathrm{m}\end{array}$ \\
\hline aff. Parakiefferiella & $\begin{array}{l}\text { Detritus }-88.3 \% \text { August } \\
\text { Algae }-10 \% \text { May } \\
\text { Fungi }-16.5 \% \text { February }\end{array}$ & $\begin{array}{l}\text { Chlorophyceae: colonial, filamentous } \\
\text { and unicellular algae } \\
\text { Cyanophyceae (Anabaena?) } \\
\text { Diatomaceae } \\
\text { Fungi (conidia and hyphas) } \\
\text { Leaf and wood fragments and detritus } \\
\text { Pollens (several types) }\end{array}$ & $\begin{array}{l}\text { Max. }-100 \mu \mathrm{m} \\
\text { Min. }-12.5 \mu \mathrm{m}\end{array}$ \\
\hline Parametriocnemus & $\begin{array}{l}\text { Detritus }-90.5 \% \\
\text { November } \\
\text { Leaf and wood fragments - } \\
21.8 \% \text { February } \\
\text { Algae }-4.6 \% \text { August }\end{array}$ & $\begin{array}{l}\text { Chlorophyceae: filamentous and } \\
\text { unicellular algae } \\
\text { Cyanophyceae (Anabaena?) } \\
\text { Desmidiaceae (Cosmarium) } \\
\text { Diatomaceae } \\
\text { Fungi (conidia and hyphas) } \\
\text { Leaf and wood fragments and detritus } \\
\text { Pollens (several types) and spores } \\
\text { Oligochaeta? }\end{array}$ & $\begin{array}{l}\text { Max. }-400 \mu \mathrm{m} \\
\text { Min. }-20 \mu \mathrm{m}\end{array}$ \\
\hline Pseudosmittia & $\begin{array}{l}\text { Detritus }-86.1 \% \\
\text { November } \\
\text { Leaf and wood fragments - } \\
34.5 \% \text { February } \\
\text { Fungi }-7.6 \% \text { November }\end{array}$ & $\begin{array}{l}\text { Chlorophyceae: filamentous and } \\
\text { unicellular algae } \\
\text { Fungi (conidia and hyphas) } \\
\text { Leaf and wood fragments and detritus } \\
\text { Pollens (several types) and spores }\end{array}$ & $\begin{array}{l}\text { Max. }-87 \mu \mathrm{m} \\
\text { Min. }-12.5 \mu \mathrm{m}\end{array}$ \\
\hline Thienemanniella & $\begin{array}{l}\text { Detritus }-92.7 \% \text { May } \\
\text { Algae }-5.4 \% \text { August } \\
\text { Fungi }-4.4 \% \text { November } \\
\text { Pollen }-3.7 \% \text { November }\end{array}$ & $\begin{array}{l}\text { Chlorophyceae: colonial, filamentous } \\
\text { and unicellular algae } \\
\text { Fungi (conidia and hyphas) } \\
\text { Leaf and wood fragments and detritus } \\
\text { Pollens (several types) and spores }\end{array}$ & $\begin{array}{l}\text { Max. }-50 \mu \mathrm{m} \\
\text { Min. }-5 \mu \mathrm{m}\end{array}$ \\
\hline
\end{tabular}


TABLE 4

Average contribution of each food item for the chironomid genera studied in the Rio da Fazenda, between August 1994 and May 1995. (Mean - arithmethic mean; SD - standard deviation.)

\begin{tabular}{|c|c|c|c|c|c|c|c|c|}
\hline & & Algae & Fungi & Pollen & \begin{tabular}{|c|}
$\begin{array}{c}\text { Leaf and } \\
\text { wood }\end{array}$ \\
\end{tabular} & \begin{tabular}{l|} 
Animal \\
remains
\end{tabular} & Detritus & Silt \\
\hline \multicolumn{9}{|l|}{ Tanypodinae } \\
\hline \multirow{2}{*}{ Ablabesmyia } & Mean & 1.82 & 1.42 & 1.62 & 2.40 & 28.46 & 61.34 & 2.92 \\
\hline & SD & 1.37 & 0.73 & 0.80 & 1.99 & 13.05 & 11.93 & 2.68 \\
\hline \multirow{2}{*}{ cf. Djalmabatista } & Mean & 3.04 & 0.94 & 2.18 & 2.15 & 32.29 & 51.72 & 7.68 \\
\hline & SD & 3.57 & 0.53 & 2.02 & 2.13 & 11.33 & 4.25 & 6.08 \\
\hline \multirow{2}{*}{ Labrundinia } & Mean & 4.49 & 1.58 & 4.66 & 4.28 & 0.49 & 83.50 & 1.02 \\
\hline & SD & 1.54 & 1.13 & 3.55 & 5.35 & 0.84 & 4.19 & 1.19 \\
\hline \multirow{2}{*}{ aff. Larsia } & Mean & 5.51 & 1.61 & 1.86 & 2.46 & 16.65 & 70.04 & 1.88 \\
\hline & SD & 5.22 & 0.87 & 0.56 & 1.23 & 3.50 & 3.83 & 1.67 \\
\hline \multirow{2}{*}{ aff. Pentaneura } & Mean & 1.13 & 1.41 & 1.28 & 2.20 & 28.16 & 60.03 & 5.78 \\
\hline & SD & 0.80 & 0.82 & 0.52 & 1.00 & 15.22 & 9.54 & 5.55 \\
\hline \multicolumn{9}{|l|}{\begin{tabular}{|l|} 
Chironominae \\
\end{tabular}} \\
\hline \multirow{2}{*}{ Chironomus } & Mean & 1.25 & 3.14 & 1.12 & 4.76 & 0.09 & 62.10 & 2.55 \\
\hline & SD & 1.78 & 1.86 & 0.89 & 4.72 & 0.15 & 36.22 & 2.41 \\
\hline \multirow{2}{*}{ Endotribelos } & Mean & 5.96 & 4.18 & 0.87 & 6.79 & 0.00 & 81.32 & 0.90 \\
\hline & SD & 5.01 & 5.70 & 0.54 & 7.21 & 0.00 & 11.97 & 0.70 \\
\hline \multirow{2}{*}{ aff. Nilothauma } & Mean & 3.55 & 29.30 & 1.53 & 6.50 & 0.00 & 59.13 & 0.00 \\
\hline & SD & 6.15 & 41.22 & 1.35 & 6.54 & 0.00 & 34.48 & 0.00 \\
\hline \multirow{2}{*}{ aff. Omisus } & Mean & 1.95 & 2.65 & 1.63 & 3.90 & 0.00 & 88.99 & 0.86 \\
\hline & SD & 1.03 & 0.88 & 0.24 & 1.72 & 0.00 & 1.29 & 0.71 \\
\hline \multirow{2}{*}{ Phaenopsectra } & Mean & 4.26 & 1.40 & 1.51 & 11.41 & 0.14 & 55.36 & 0.92 \\
\hline & SD & 3.86 & 1.24 & 0.97 & 12.60 & 0.14 & 34.09 & 0.58 \\
\hline \multirow{2}{*}{ Polypedilum } & Mean & 7.45 & 3.82 & 1.70 & 7.12 & 0.00 & 79.04 & 0.88 \\
\hline & SD & 7.01 & 1.79 & 0.71 & 2.55 & 0.00 & 8.86 & 0.81 \\
\hline \multirow{2}{*}{ Rheotanytarsus } & Mean & 1.65 & 2.64 & 2.27 & 0.95 & 0.00 & 90.67 & 1.83 \\
\hline & SD & 0.96 & 2.09 & 0.96 & 0.39 & 0.00 & 2.10 & 1.11 \\
\hline \multirow{2}{*}{ Stempellinella } & Mean & 0.89 & 3.53 & 1.53 & 1.97 & 0.00 & 91.02 & 1.07 \\
\hline & SD & 0.67 & 1.79 & 0.62 & 0.66 & 0.00 & 2.38 & 0.42 \\
\hline \multirow{2}{*}{ Stenochironomus } & Mean & 8.03 & 0.13 & 0.05 & 87.23 & 0.00 & 4.57 & 0.00 \\
\hline & SD & 4.16 & 0.13 & 0.09 & 6.41 & 0.00 & 6.27 & 0.00 \\
\hline \multirow{2}{*}{ Tanytarsus } & Mean & 1.15 & 1.86 & 1.63 & 2.32 & 0.00 & 91.93 & 1.12 \\
\hline & SD & 0.37 & 0.45 & 0.62 & 0.84 & 0.00 & 1.28 & 0.60 \\
\hline \multirow{2}{*}{ aff. Tribelos } & Mean & 3.07 & 1.96 & 1.30 & 13.45 & 0.00 & 79.11 & 1.12 \\
\hline & SD & 0.52 & 2.33 & 0.61 & 9.49 & 0.00 & 7.43 & 0.66 \\
\hline \multirow{2}{*}{ Chironomini tipo 1} & Mean & 7.57 & 2.15 & 1.39 & 3.85 & 0.04 & 83.95 & 1.05 \\
\hline & SD & 5.99 & 1.30 & 0.48 & 0.79 & 0.06 & 5.25 & 0.42 \\
\hline \multicolumn{9}{|l|}{ Orthocladiinae } \\
\hline \multirow{2}{*}{ Corynoneura } & Mean & 0.40 & 2.20 & 1.93 & 1.34 & 0.00 & 92.46 & 1.68 \\
\hline & SD & 0.69 & 2.09 & 0.70 & 2.12 & 0.00 & 5.13 & 1.80 \\
\hline \multirow{2}{*}{ Lopescladius } & Mean & 0.00 & 1.12 & 1.79 & 0.52 & 0.00 & 96.07 & 0.50 \\
\hline & SD & 0.00 & 0.51 & 0.60 & 0.34 & 0.00 & 0.55 & 0.22 \\
\hline \multirow{2}{*}{ Nanocladius } & Mean & 6.71 & 3.98 & 3.19 & 2.49 & 0.00 & 81.92 & 1.71 \\
\hline & SD & 5.51 & 4.79 & 0.87 & 1.46 & 0.00 & 7.57 & 0.66 \\
\hline
\end{tabular}


TABLE 4 (Continued.)

\begin{tabular}{|c|c|c|c|c|c|c|c|c|}
\hline & & Algae & Fungi & Pollen & \begin{tabular}{|c|}
$\begin{array}{c}\text { Leaf and } \\
\text { wood }\end{array}$ \\
\end{tabular} & $\begin{array}{c}\text { Animal } \\
\text { remains }\end{array}$ & Detritus & Silt \\
\hline \multicolumn{9}{|l|}{ Orthocladiinae } \\
\hline \multirow{2}{*}{ aff. Parakiefferiella } & Mean & 7.04 & 5.76 & 2.01 & 1.92 & 0.00 & 81.18 & 2.09 \\
\hline & $\mathrm{SD}$ & 7.55 & 6.31 & 1.23 & 0.84 & 0.00 & 6.01 & 0.82 \\
\hline \multirow{2}{*}{ Parametriocnemus } & Mean & 2.31 & 2.30 & 1.67 & 8.75 & 0.50 & 82.95 & 1.52 \\
\hline & SD & 1.53 & 0.93 & 0.15 & 7.87 & 0.61 & 8.52 & 0.47 \\
\hline \multirow{2}{*}{ Pseudosmittia } & Mean & 2.06 & 3.57 & 1.80 & 18.77 & 0.00 & 73.15 & 0.66 \\
\hline & SD & 1.93 & 2.43 & 1.16 & 11.55 & 0.00 & 9.49 & 0.50 \\
\hline \multirow{2}{*}{ Thienemanniella } & Mean & 2.74 & 2.05 & 2.74 & 1.15 & 0.00 & 90.83 & 0.47 \\
\hline & SD & 2.23 & 1.39 & 0.89 & 1.66 & 0.00 & 1.29 & 0.46 \\
\hline
\end{tabular}

TABLE 5

Chi-square values found for the gut contents of chironomid larvae from the Rio da Fazenda,

Parque Nacional da Tijuca, RJ, between August 1994 and May 1995 (p < 0.05).

\begin{tabular}{|l|c|}
\hline \multicolumn{1}{|c|}{ Taxa } & Chi-square values $\left(\boldsymbol{X}^{\mathbf{2}}\right)$ \\
\hline Ablabesmyia & 56.99 \\
\hline cf. Djalmabatista & 70.35 \\
\hline Labrundinia & 55.16 \\
\hline aff. Larsia & 34.50 \\
\hline aff. Pentaneura & 67.16 \\
\hline Chironomus & 23.06 \\
\hline Endotribelos & 89.11 \\
\hline aff. Nilothauma & 386.08 \\
\hline aff. Omisus & 6.50 \\
\hline Phaenopsectra & 46.93 \\
\hline Polypedilum & 41.63 \\
\hline Rheotanytarsus & 14.02 \\
\hline Stempellinella & 8.42 \\
\hline Stenochironomus & 46.07 \\
\hline Tanytarsus & 4.43 \\
\hline aff. Tribelos & 43.67 \\
\hline Chironomini sp. 1 & 25.81 \\
\hline Corynoneura & 36.03 \\
\hline Lopescladius & 2.97 \\
\hline Nanocladius & 49.38 \\
\hline aff. Parakiefferiella & 67.61 \\
\hline Parametriocnemus & 40.98 \\
\hline Pseudosmittia & 51.74 \\
\hline Thienemanniella & 23.65 \\
\hline & \\
\hline
\end{tabular}




\section{DISCUSSION}

Gut content analysis showed that 16 out of the 24 chironomid genera studied in this work showed a change in their feeding habits among the four months studied. This variation in the diet may suggest that these groups present a low degree of selectivity, having more generalist habits. According to Berg (1995) there are few chironomid species that present nutritional selectivity, the great majority being generalists and opportunistic.

The same pattern was confirmed by Nessimian (1997), studying the functional categorization of macroinvertebrates in a sand dune marsh in Maricá (State of Rio de Janeiro) found that most species had generalist and opportunistic habits, feeding on what was available at that moment. Nessimian \& Sanseverino (1998), studying the diet of chironomid larvae in a stream in the mountain region of the State of Rio de Janeiro, registered that the composition of the gut contents changed according to the type of microhabitat (e.g., type of substrate) where the larvae were found. Also, they observed differences among samples from the same microhabitats in different seasons, as a result of changes in the type of available substrate.

Detritus was the main food item found in most of the larvae studied. According to Berg (1995), it is not unusual for this item to represent $50 \%-70 \%$ of the gut contents of chironomids. Nessimian \& Sanseverino (1998) found detritus as the most abundant item in the diet of Chironominae and Orthocladiinae genera from the Paquequer River (Teresópolis, State of Rio de Janeiro), together with a high concentration of pollen and spores. In Rio da Fazenda, the most important items, after detritus, were leaf and wood fragments as well as fungi in the diet of the Chironominae and Orthocladiinae subfamilies, while animal remains were found in Tanypodinae.

The highest diversity of food items was observed in the Tanypodinae. Several genera of this subfamily presented algae, fungi, pollen, leaf and wood fragments, and detritus, but also many Chironominae and Orthocladiinae genera as food items. Pinder (1986) points out that the Tanypodinae larvae have a quite diverse diet among animals and plants items. The occurrence of some genera in the gut contents of these chironomids is due to their availability in the habitat and can also be related to prey size and the predator's development stage. Labrundinia was the only genus in this subfamily with non-animal material in the gut contents. This genus is reported by Nessimian et al. (1999), working in a sand dune marsh in the coast of Rio de Janeiro, as feeding on animal sources (chironomid larvae, Cladocera, and Copepoda), mainly in summer and spring (dry season), and diatoms in the autumn and winter (rainy season). The absence of animal matter in the gut contents of Labrundinia larvae in the Rio da Fazenda might reflect the small size of the sampled individuals. This fact agrees with Oliver (1971) and Cummins (1973), which recorded that larvae at different developmental stages often have different diets.

Among Chironominae and Orthocladiinae larvae the greatest change was detected in Nilothauma $\left(\mathrm{X}^{2}=386.08\right)$. This genus showed only fungi in the gut contents of the individuals analyzed in August, while the highest diversity of items was found in the individuals analyzed in May of 1995, when detritus, leaf and wood fragments, fungi, and algae was observed in their gut contents. Stenochironomus was the only genus, which fed mainly on leaf and wood fragments, feeding both on leaf cells and wood fibers. These larvae are considered by many authors to be truly xylophagous (e.g., Cranston \& Oliver, 1988; Berg, 1995). Epler (1995) points out that Stenochironomus larvae are miners of submerged leaves and wood fragments.

The particle analysis of the size of what was ingested by the larvae revealed that, with the exception of the Tanypodinae, which might be classified as predators since they feed upon animal material frequently higher than $1 \mathrm{~mm}$, most chironomid larvae are classified as collectors, feeding upon fine particulate organic matter (FPOM). Also, according to Cummins (1974), FPOM can be divided in three categories: MPOM - mean particulate organic matter, ranging from 250 to $1000 \mu \mathrm{m}$; SPOM - small particulate organic matter, ranging from 75 to $250 \mu \mathrm{m}$; and VPOM - very fine particulate organic matter, ranging from 0.5 to 75 $\mu \mathrm{m}$. According to this classification, most of the Chironominae and Orthocladiinae genera showed SPOM in their gut contents.

Two groups of collectors were found in the Rio da Fazenda: the collector-gatherers and the collectorsfilterers. In this latter group we can include Nilothauma, Rheotanytarsus, Corynoneura, Lopescladius, and 
Thienemanniella, which frequently ingested particles smaller than $75 \mu \mathrm{m}$.

On the other hand Chironomus, Phaenopsectra, aff. Tribelos, and Parametriocnemus showed MPOM in their gut contents, and might be considered besides collector-gatherers, as possible scrapers and shredders, since the capture and food ingestion can involve more than one feed mode, and in this genera leaf and wood fragments, algae and other materials often higher than $250 \mu \mathrm{m}$ were found. Stenochironomus might be considered as a true shredder, since it presented fibers and leaf and wood fragments as the main food source.

Although the functional group categories are based partially in the morphology of the species, there is considerable flexibility in the mode of feeding among chironomids. Many factors, such as larval size, food quality and type of sediment might influence the larval feeding behavior (Berg, 1995). In addition to that, the change in the larval diet might also reflect the differences observed in the structure of the Rio da Fazenda, which determines changes in the structure and composition of the substrates regarding the quality of the available resources. According to Nessimian \& Sanseverino (1998), changes in the feeding habits of chironomids are dependent, to a great extension, on environmental conditions.

Acknowledgments - The authors acknowledge CNPq and FAPERJ for financial support and for the grant provided to the first author by CAPES.

\section{REFERENCES}

BERG, H. B., 1995, Larval food and feeding behaviour, pp. 136168. In: P. D. Armitage, P. S. Cranston \& L. C. V. Pinder (eds.), The Chironomidae: biology and ecology of non-biting midges. Chapman \& Hall, London, 584p.

COFFMAN, W. P. \& FERRINGTON, L. C., 1996, Chironomidae, pp: 635-754. In: K. W. Merrit \& R. W. Cummins (eds.), An introduction of aquatic insects of North America. 3. ed. Kendall Hunt Publishing Co., Dubuque, xii $+862 p$.

CRANSTON, P. S., OLIVER, D. R. \& SÆTHER, O. A., 1983, The larvae of Orthocladiinae (Diptera: Chironomidae) of the Holartic region - keys and diagnoses. In: T. Wiederholm (ed.), Chironomidae of the Holartic region. Keys and diagnose. Part I: Larvae. Ent. Scan. Suppl., 19: 149-291.

CRANSTON, P. S. \& OLIVER, D. R., 1988, Aquatic xylophagous Orthocladiinae - systematics and ecology. Spixiana Suppl., 14: 143-154.

CUMMINS, K. W. \& KLUG, M. J., 1979, Feeding ecology on stream invertebrates. Ann. Ver. Ecol. Syst., 10: 147-172.

CUMMINS, K. W., 1973, Trophic relations of aquatic insects. Annual Review of Entomology, 18: 183-206.

CUMMINS, K. W., 1974, Structure and functions of stream ecosystems. Bioscience, 24: 631-641.

DRUMMOND, J. A., 1997, Devastação e preservação ambiental: os parques nacionais do Estado do Rio de Janeiro. (Coleção Antropologia e Ciência Política, 2). EDUFF, Niterói, RJ, 306p.

EPLER, J. H., 1995, Identification manual for the larvae Chironomidae (Diptera) of Florida. Department of Environmental Protection, Division of Water Facilities, Tallahassee, 250p.

HIRABAYASHI, K. \& WOTTON, R. S., 1998, Organic matter processing by chironomid larvae (Diptera: Chironomidae) Hydrobiologia, 382: 151-159.

MERRIT, K. W., CUMMINS, R. W. \& BURTON, T. M., 1984, The role of Aquatic insects in the processing and cycling of nutrients, pp: 134-163. In: V. H. Resh \& D. M. Rosenberg (eds.), The ecology of aquatic insects. Praeger Publishers, New York, $\mathrm{xi}+625 \mathrm{p}$.

NESSIMIAN, J. L. \& SANSEVERINO, A. M., 1998, Trophic functional categorization of the chironomid larvae (Diptera: Chironomidae) in a first-order stream at the mountain region of Rio de Janeiro State, Brazil. Verh. Internat. Verein. Limnol., 26(IV): 2115-2119.

NESSIMIAN, J. L., 1997, Categorização funcional de macroinvertebrados de um brejo de dunas no Estado do Rio de Janeiro. Rev. Brasil. Biol., 57(1): 135-145.

NESSIMIAN, J. L., SANSEVERINO, A. M. \& OLIVEIRA, A. L. H., 1999, Relações tróficas de larvas de Chironomidae (Diptera) e sua importância na rede alimentar em um brejo no litoral do Estado do Rio de Janeiro. Revta. Brasil. Entomol., 43(1/2): 47-53.

OLIVER, D. R., 1971, Life history of the Chironomidae. Ann. Rev. Entomol., 12: 211-230.

PINDER, L. C. V. \& REISS, F., 1983, The larvae of Chironominae (Diptera: Chironomidae) of the Holartic region. Keys and diagnoses. In: T. Wiederholm (ed.), Chironomidae of the Holartic region. Keys and diagnose. Part I: Larvae. Ent. Scan. Suppl., 19: 293-435.

PINDER, L. C. V., 1986, Biology of freshwater Chironomidae. Ann. Rev. Entomol., 31: 1-23.

TOKESHI, M., 1995, Production ecology, pp: 269-296. In: P. D. Armitage, P. S. Cranston \& L. C. V. Pinder. The Chironomidae. Biology and ecology of non-biting midges. Chapman \& Hall, London, 584p.

TRIVINHO-STRIXINO, S. \& STRIXINO, G., 1995, Larvas de Chironomidae (Diptera) do Estado de São Paulo: Guia de identificação e diagnose dos gêneros. PPGE-RN, Universidade Federal de São Carlos, SP, 227p. 